\title{
Synthesis and Reactivity of Diborane(4)yl Complexes
}

Holger Braunschweig ${ }^{\mathrm{a}}$ and Margot Koster ${ }^{\mathrm{b}}$

a Department of Chemistry, Imperial College of Science, Technology, and Medicine, South Kensington, London, SW7 2AY, UK

${ }^{\mathrm{b}}$ Institut für Anorganische Chemie der Technischen Hochschule Templergraben 55, D-52056 Aachen, Germany

Reprint requests to Dr. H. Braunschweig. Fax: ++44 (207) 594-5900.

E-mail: h.braunschweig@ic.ac.uk

Z. Naturforsch. 57 b, 483-487 (2002); received December 19, 2001

Boranes, Diboranes(4), Boryl Complexes

Reaction of various 1,2-dihalodiboranes (4) with $\mathrm{Na}\left[\left(\eta^{5}-\mathrm{C}_{5} \mathrm{H}_{4} \mathrm{R}\right) \mathrm{M}(\mathrm{CO})_{3}\right]$ yielded the diborane(4)yl complexes $\left[\mathrm{X}\left(\mathrm{Me}_{2} \mathrm{~N}\right) \mathrm{B}-\mathrm{B}\left(\mathrm{NMe}_{2}\right)\left\{\eta^{5}-\left(\mathrm{C}_{5} \mathrm{H}_{4} \mathrm{R}\right) \mathrm{M}(\mathrm{CO})_{3}\right\}\right](\mathbf{5}, \mathrm{X}=\mathrm{Cl}, \mathrm{R}=\mathrm{H}$, $\mathrm{M}=\mathrm{Mo} ; \mathbf{6}, \mathrm{X}=\mathrm{Br}, \mathrm{R}=\mathrm{Me}, \mathrm{M}=\mathrm{Mo} ; \mathbf{7}, \mathrm{X}=\mathrm{Br}, \mathrm{R}=\mathrm{H}, \mathrm{M}=\mathrm{W} ; \mathbf{8}, \mathrm{X}=\mathrm{Br}, \mathrm{R}=$ $\mathrm{Me}, \mathrm{M}=\mathrm{W})$ and $\left[\mathrm{Cl}\left(\mathrm{C}_{4} \mathrm{H}_{8} \mathrm{~N}\right) \mathrm{B}-\mathrm{B}\left(\mathrm{NC}_{4} \mathrm{H}_{8}\right)\left\{\eta^{5}-\left(\mathrm{C}_{5} \mathrm{H}_{5}\right) \mathrm{W}(\mathrm{CO})_{3}\right\}\right]$ (9). The reactivity of diborane $(4) y l$ complexes of the type $\left[\mathrm{X}\left(\mathrm{Me}_{2} \mathrm{~N}\right) \mathrm{B}-\mathrm{B}\left(\mathrm{NMe}_{2}\right)\left\{\eta^{5}-\left(\mathrm{C}_{5} \mathrm{H}_{5}\right) \mathrm{M}(\mathrm{CO})_{n}\right\}\right](\mathrm{X}=\mathrm{Cl}, \mathrm{Br}$; $\left.\mathrm{M}(\mathrm{CO})_{n}=\mathrm{Fe}(\mathrm{CO})_{2}, \mathrm{Mo}(\mathrm{CO})_{3}\right)$ with respect to substitution of $\mathrm{X}$ and $\mathrm{CO}$, respectively, was investigated. The novel derivatives $\left[\mathrm{MeO}\left(\mathrm{Me}_{2} \mathrm{~N}\right) \mathrm{B}-\mathrm{B}\left(\mathrm{NMe}_{2}\right)\left\{\eta^{5}-\left(\mathrm{C}_{5} \mathrm{H}_{5}\right) \mathrm{Mo}(\mathrm{CO})_{3}\right\}\right](\mathbf{1 0})$, $\left[\mathrm{MeO}\left(\mathrm{Me}_{2} \mathrm{~N}\right) \mathrm{B}-\mathrm{B}\left(\mathrm{NMe}_{2}\right)\left\{\eta^{5}-\left(\mathrm{C}_{5} \mathrm{H}_{5}\right) \mathrm{Fe}(\mathrm{CO})_{2}\right\}\right](\mathbf{1 1})$, and $\left[\mathrm{Br}\left(\mathrm{Me}_{2} \mathrm{~N}\right) \mathrm{B}-\mathrm{B}\left(\mathrm{NMe}_{2}\right)\left\{\eta^{5}-\left(\mathrm{C}_{5} \mathrm{H}_{5}\right)-\right.\right.$ $\left.\left.\mathrm{Mo}(\mathrm{CO})_{2}\left(\mathrm{PMe}_{3}\right)\right\}\right](\mathbf{1 2})$ were obtained. All compounds were fully characterised in solution by NMR spectroscopy and by elemental analysis.

\section{Introduction}

Over the past decade transition metal complexes of boron have become established as the fourth class of compounds made up by direct metal-boron interactions. In contrast to the other three major groups in this area i. e. borides, metallaboranes, and $\pi$-complexes with boron-containing ligands, transition metal complexes of boron are characterised by electron-precise two-centre two-electron bonds between boron and the metal centre [1]. Most of these compounds are derived from monoboranes, but diboranes(4) also play an important role in the chemistry of these complexes. With strongly electronegative groups $\mathrm{RO}$ [2] and $\mathrm{F}^{-}$[3] as substituents to both boron atoms the corresponding diboranes(4) generally react with cleavage

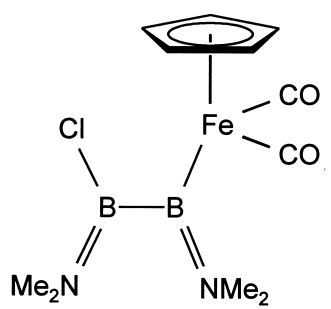

(1)

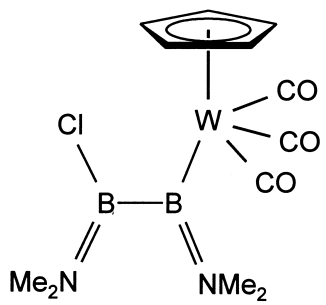

(2)

of the boron-boron bond and oxidative addition to suitable metal centres, thus forming products with one to three borylligands. The use of 1,2diaminodihalodiboranes(4) and anionic transition metal complexes, however, has led to the first diborane (4)yl complexes $\left[\mathrm{Cl}\left(\mathrm{Me}_{2} \mathrm{~N}\right) \mathrm{B}-\mathrm{B}\left(\mathrm{NMe}_{2}\right)\right.$ $\left.\left\{\eta^{5}-\left(\mathrm{C}_{5} \mathrm{H}_{5}\right) \mathrm{M}(\mathrm{CO})_{n}\right\}\right] \quad\left(\mathbf{1}, \mathrm{M}(\mathrm{CO})_{n}=\mathrm{Fe}(\mathrm{CO})_{2}\right.$; 2, $\left.\mathrm{M}(\mathrm{CO})_{n}=\mathrm{W}(\mathrm{CO})_{3}\right)$ [4], and $\left[\mathrm{Br}\left(\mathrm{Me}_{2} \mathrm{~N}\right) \mathrm{B}-\right.$ $\left.\mathrm{B}\left(\mathrm{NMe}_{2}\right)\left\{\eta^{5}-\left(\mathrm{C}_{5} \mathrm{H}_{5}\right) \mathrm{M}(\mathrm{CO})_{n}\right\}\right] \quad\left(3, \quad \mathrm{M}(\mathrm{CO})_{n}=\right.$ $\left.\mathrm{Ru}(\mathrm{CO})_{2} ; 4, \mathrm{M}(\mathrm{CO})_{n}=\mathrm{Mo}(\mathrm{CO})_{3}\right)$ [5], which were obtained via salt elimination reactions (Fig. 1). Complexes of this type are still rare and are restricted to $\mathbf{1}-\mathbf{4}$ and two further examples $\left[\mathrm{Cl}\left(\mathrm{R}_{2} \mathrm{~N}\right) \mathrm{B}-\mathrm{B}\left(\mathrm{NR}_{2}\right)\left\{\eta^{5}-\left(\mathrm{C}_{5} \mathrm{H}_{5}\right) \mathrm{Fe}(\mathrm{CO})_{2}\right\}\right]$ $\left(\mathrm{NR}_{2}=\mathrm{NC}_{4} \mathrm{H}_{8}, \mathrm{NC}_{5} \mathrm{H}_{10}\right)$ [6]. Although the reactivity of related boryl complexes $\mathrm{L}_{x} \mathrm{M}-\mathrm{BR}_{2}$ is well established [1], there is no informa-

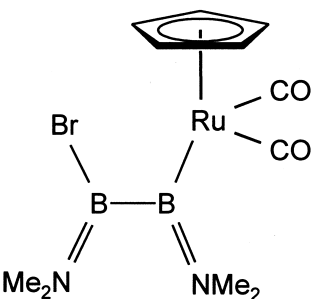

(3)

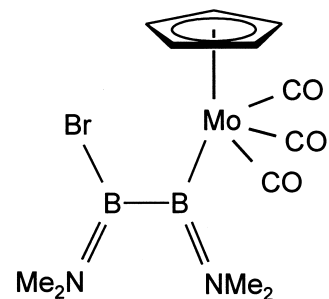

(4) 
<smiles></smiles>

\section{$\mathrm{Na}\left[\left(\eta^{5}-\mathrm{C}_{5} \mathrm{H}_{4} \mathrm{R}\right) \mathrm{M}(\mathrm{CO})_{3}\right]$}

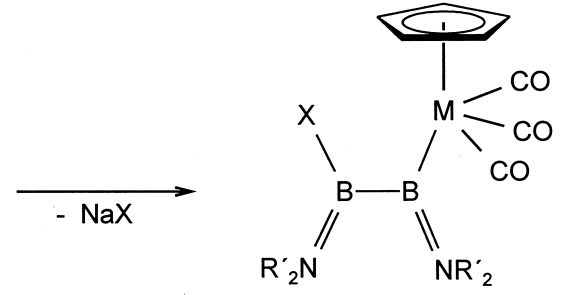

5; $X=\mathrm{Cl}, \mathrm{M}=\mathrm{Mo}, \mathrm{R}=\mathrm{H}, \quad \mathrm{R}_{2}{ }^{\prime} \mathrm{N}=\mathrm{Me}_{2} \mathrm{~N}$

6: $X=B r, M=M o, R=M e, R_{2}{ }^{\prime} N=M_{2} N$

7; $X=B r, M=W, \quad R=H, \quad R_{2}{ }^{\prime} N=M e_{2} N$

8: $X=B r, M=W, R=M e, R_{2}{ }^{\prime} N=M_{2} N$

9: $X=C l, M=W, \quad R=H, \quad R_{2}{ }^{\prime} N={ }_{N}^{N}$

the inspection of the reaction mixtures provided no evidence for the formation of boryloxy compounds as possible side products. All new complexes were characterised in solution by IR and multinuclear NMR spectroscopy. They show characteristically deshielded ${ }^{11} \mathrm{~B}$ NMR resonances $\left({ }^{11} \mathrm{~B}=65.4(5)\right.$, $65.9(6), 62.7(7), 63.2(8), 60.2(9))$ for the metal coordinated boron atoms with respect to those of the starting materials, while the resonances for the halide substituted boron atoms range from $\delta^{11} \mathrm{~B}=$ 38.0 to 40.0 , thus matching those of the non-coordinated diboranes(4). The complexes 5 - 8 show four signals for the dimethylamino ligands in the ${ }^{1} \mathrm{H}$ and ${ }^{13} \mathrm{C}$ NMR spectra, thus giving clear evidence for a restricted rotation with respect to both boron-nitrogen double bonds $[4,5]$. Corresponding ${ }^{1} \mathrm{H}$ NMR signals for the more complex pyrrolidino groups in 9 could not be resolved. The presence of eight signals in the ${ }^{13} \mathrm{C}$ NMR spectrum ranging from $\delta=25.46$ to 55.76, however, indicates the same situation with respect to the two boron-nitrogen bonds. All five compounds show three very similar $\mathrm{CO}$-stretching frequencies in the IR spectra ranging from $\nu=1883$ to $1989 \mathrm{~cm}^{-1}$, which match those of the structurally characterised tungsten diborane(4)yl complex 2 ( $\nu=$ 1892,1908 , and $1988 \mathrm{~cm}^{-1}$ ), thus giving no evidence for a metal-boron $\pi$-backbonding $[4,5]$.

\section{Reactivity Studies}

In order to investigate some reactions of diboran(4)yl complexes [Cl( $\left.\mathrm{Me}_{2} \mathrm{~N}\right) \mathrm{B}-\mathrm{B}\left(\mathrm{NMe}_{2}\right)$ $\left.\left\{\eta^{5}-\left(\mathrm{C}_{5} \mathrm{H}_{5}\right) \mathrm{Fe}(\mathrm{CO})_{2}\right\}\right]$ (1) [4] and $\left[\mathrm{Br}\left(\mathrm{Me}_{2} \mathrm{~N}\right) \mathrm{B}-\right.$ $\left.\mathrm{B}\left(\mathrm{NMe}_{2}\right)\left\{\eta^{5}-\left(\mathrm{C}_{5} \mathrm{H}_{5}\right) \mathrm{Mo}(\mathrm{CO})_{3}\right\}\right]$ (4) [5] were cho- 


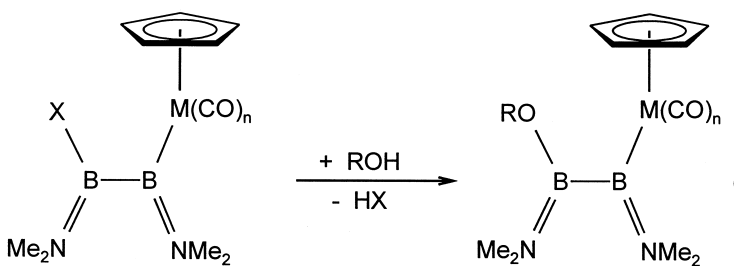

1: $\mathrm{X}=\mathrm{Cl}, \mathrm{M}(\mathrm{CO})_{\mathrm{n}}=\mathrm{Fe}(\mathrm{CO})_{2}$

4: $\mathrm{X}=\mathrm{Br}, \mathrm{M}(\mathrm{CO})_{\mathrm{n}}=\mathrm{Mo}(\mathrm{CO})_{3}$
(2)<smiles></smiles>

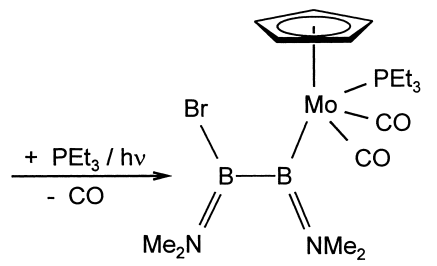

14

In the IR spectrum the $\mathrm{CO}$ stretching frequencies range between 1932 and $1812 \mathrm{~cm}^{-1}$, thus displaying the expected red shift due to substitution of one carbonyl group by triethylphosphane.

\section{Experimental Section}

NMR: Varian Unity $500\left({ }^{1} \mathrm{H},{ }^{11} \mathrm{~B},{ }^{13} \mathrm{C},{ }^{31} \mathrm{P}\right)$ in $\mathrm{C}_{6} \mathrm{D}_{6}$ with usual standards. IR: Perkin-Elmer FT-IR 1720. Elemental analyses $(\mathrm{C}, \mathrm{H}, \mathrm{N})$ : Carlo-Erba elemental analyzer, model 1106.

5: $0.50 \mathrm{~g}(1.76 \mathrm{mmol})$ of $\mathrm{K}\left[\left\{\eta^{5}-\left(\mathrm{C}_{5} \mathrm{H}_{5}\right) \mathrm{Mo}(\mathrm{CO})_{3}\right\}\right]$ [9] suspended in $10 \mathrm{ml}$ of benzene is treated with $0.32 \mathrm{~g}$ $(1.76 \mathrm{mmol})$ of $\mathrm{B}_{2}\left(\mathrm{NMe}_{2}\right)_{2} \mathrm{Cl}_{2}[10]$ and the mixture is stirred for $6 \mathrm{~d}$ at ambient temperature. All volatiles are removed under high vacuum, and the residue is treated with $35 \mathrm{ml}$ of hexane. After filtration $0.35 \mathrm{~g}(51.0 \%)$ of 5 is obtained at $-30^{\circ} \mathrm{C}$ as a yellow solid. $-\mathrm{IR}$ (toluene): $\nu=$ 1982, 1900, 1887 (CO) $\mathrm{cm}^{-1}$. - ${ }^{1} \mathrm{H}$ NMR (499.843 MHz): $\delta=2.41,2.53,2.64,2.84\left(4 \mathrm{~s}, 12 \mathrm{H}, \mathrm{NMe}_{2}\right), 4.98(\mathrm{~s}, 5$ $\left.\mathrm{H}, \mathrm{C}_{5} \mathrm{H}_{5}\right) .-{ }^{11} \mathrm{~B}$ NMR $(160.34 \mathrm{MHz}): \delta=38.0(\mathrm{BCl})$, 65.4 (BMo). $-{ }^{13} \mathrm{C}\left\{{ }^{1} \mathrm{H}\right\}$ NMR (125.697 MHz): $\delta=$ 36.90, 41.16, 42.39, 47.72 $\left(\mathrm{NMe}_{2}\right), 93.64\left(\mathrm{C}_{5} \mathrm{H}_{5}\right), 224.63$, 227.68, 233.63 (CO). $-\mathrm{C}_{12} \mathrm{H}_{17} \mathrm{~B}_{2} \mathrm{ClMoN}_{2} \mathrm{O}_{3}$ (390.29): calcd. C 36.93, H 4.39, N 7.18; found C 36.68, H 4.47, N 7.19.

6: As described for 5, $0.99 \mathrm{~g}(3.32 \mathrm{mmol})$ of $\mathrm{K}\left[\left\{\eta^{5}-\left(\mathrm{C}_{5} \mathrm{H}_{4} \mathrm{Me}\right) \mathrm{Mo}(\mathrm{CO})_{3}\right\}\right]$ [9] is treated with $0.90 \mathrm{~g}$ (3.34 mmol) of $\mathrm{B}_{2}\left(\mathrm{NMe}_{2}\right)_{2} \mathrm{Br}_{2}$ [11] to give $0.78 \mathrm{~g}(52.0 \%)$ 6 as a red solid. - IR (hexane): $\nu=1979,1898,1883$ (CO) $\mathrm{cm}^{-1}$. - ${ }^{1} \mathrm{H}$ NMR: $\delta=1.74$ (s, $\left.3 \mathrm{H}, \mathrm{C}_{5} \mathrm{H}_{4} M e\right), 2.48,2.64$, 2.66, $2.84\left(4 \mathrm{~s}, 12 \mathrm{H}, \mathrm{NMe}_{2}\right), 4.97\left(\mathrm{~m}, 4 \mathrm{H}, \mathrm{C}_{5} \mathrm{H}_{4} \mathrm{Me}\right)$. ${ }^{11}$ B NMR: $\delta=38.2(\mathrm{BBr}), 65.9(\mathrm{BMo}) .-{ }^{13} \mathrm{C}\left\{{ }^{1} \mathrm{H}\right\}$ NMR: $\delta=14.06\left(\mathrm{C}_{5} \mathrm{H}_{4} \mathrm{Me}\right), 39.46,41.98,42.44,47.76\left(\mathrm{NMe}_{2}\right)$, 92.83, 92.84, 94.19, 94.68, $111.94\left(C 5 \mathrm{H}_{4} \mathrm{Me}\right), 224.97$, 228.32, $234.11(\mathrm{CO}) .-\mathrm{C}_{13} \mathrm{H}_{19} \mathrm{~B}_{2} \mathrm{BrMoN}_{2} \mathrm{O}_{3}$ (448.77): calcd. C 34.79, H 4.27, N 6.24; found C 34.57, H 4.18, N 5.67.

7: As described for 5, $0.86 \mathrm{~g}$ (2.31 mmol) of $\mathrm{K}\left[\left\{\eta^{5}-\right.\right.$ $\left.\left(\mathrm{C}_{5} \mathrm{H}_{5}\right) \mathrm{W}(\mathrm{CO})_{3}\right\}$ ] [9] is treated with $0.61 \mathrm{~g}(2.31 \mathrm{mmol})$ of $\mathrm{B}_{2}\left(\mathrm{NMe}_{2}\right)_{2} \mathrm{Br}_{2}$ [11] to give $0.51 \mathrm{~g}(42.4 \%)$ of 7 as a yellow solid. - IR (hexane): $\nu=1989,1909,1892(\mathrm{CO})$ $\mathrm{cm}^{-1} .{ }^{1} \mathrm{H}$ NMR: $\delta=2.42,2.62,2.68,2.87(4 \mathrm{~s}, 12$ $\left.\mathrm{H}, \mathrm{NMe}_{2}\right), 4.96\left(\mathrm{~s}, 5 \mathrm{H}, \mathrm{C}_{5} \mathrm{H}_{5}\right) .-{ }^{11} \mathrm{~B}$ NMR: $\delta=40.0$ 
(BBr), $62.7(\mathrm{BW}) .-{ }^{13} \mathrm{C}\left\{{ }^{1} \mathrm{H}\right\}$ NMR (125.697 MHz): $\delta=$ 39.32, 41.30, 41.93, 43.08 $\left(\mathrm{NMe}_{2}\right), 92.40\left(\mathrm{C}_{5} \mathrm{H}_{5}\right), 216.17$, 219.23, 222.77 (CO). - $\mathrm{C}_{12} \mathrm{H}_{17} \mathrm{~B}_{2} \mathrm{BrWN}_{2} \mathrm{O}_{3}$ (522.65): calcd. C 27.58, H 3.28, N 5.36; found C 27.12, H 3.24, N 4.87 .

8: As described for 5, $2.13 \mathrm{~g}(5.53 \mathrm{mmol})$ of $\mathrm{K}\left[\left\{\eta^{5}-\left(\mathrm{C}_{5} \mathrm{H}_{4} \mathrm{Me}\right) \mathrm{W}(\mathrm{CO})_{3}\right\}\right]$ [9] is treated with $1.49 \mathrm{~g}$ $(5.53 \mathrm{mmol})$ of $\mathrm{B}_{2}\left(\mathrm{NMe}_{2}\right)_{2} \mathrm{Br}_{2}$ [11] to give $0.37 \mathrm{~g}(12.5 \%)$ of 8 as a yellow solid. - IR (hexane): $\nu=1987,1907,1889$ (CO) $\mathrm{cm}^{-1} .-{ }^{1} \mathrm{H}$ NMR: $\delta=1.77\left(\mathrm{~s}, 3 \mathrm{H}, \mathrm{C}_{5} \mathrm{H}_{4} M e\right), 2.47$, 2.61, 2.66, $2.89\left(4 \mathrm{~s}, 12 \mathrm{H}, \mathrm{NMe}_{2}\right), 5.00\left(\mathrm{~m}, 4 \mathrm{H}, \mathrm{C}_{5} \mathrm{H}_{4} \mathrm{Me}\right)$. ${ }_{-}^{11} \mathrm{~B}$ NMR: $\delta=40.0(\mathrm{BBr}), 63.2(\mathrm{BW}) .-{ }^{13} \mathrm{C}\left\{{ }^{1} \mathrm{H}\right\}$ NMR: $\delta=13.88\left(\mathrm{C}_{5} \mathrm{H}_{4} \mathrm{Me}\right), 39.38,41.99,43.10,48.08\left(\mathrm{NMe}_{2}\right)$, 90.80, 91.12, 93.30, 93.57, $110.93\left(\right.$ C $\left.5 \mathrm{H}_{4} \mathrm{Me}\right), 216.81$, 219.95, $233.73(\mathrm{CO}) .-\mathrm{C}_{13} \mathrm{H}_{19} \mathrm{~B}_{2} \mathrm{BrWN}_{2} \mathrm{O}_{3}$ (536.68): calcd. C 29.09, H 3.57, N 5.22; found C 28.62, H 3.61, N 5.03 .

9: As described for $5,1.97 \mathrm{~g}(5.30 \mathrm{mmol})$ of $\mathrm{K}\left[\left\{\eta^{5}-\right.\right.$ $\left.\left.\left(\mathrm{C}_{5} \mathrm{H}_{5}\right) \mathrm{W}(\mathrm{CO})_{3}\right\}\right]$ [9] is treated with $1.23 \mathrm{~g}(5.30 \mathrm{mmol})$ of $\mathrm{B}_{2}\left(\mathrm{NC}_{4} \mathrm{H}_{8}\right)_{2} \mathrm{Cl}_{2}[6,12]$ to give $0.23 \mathrm{~g}(8.1 \%)$ of 9 as a yellow solid. - IR (toluene): $\nu=1985,1900,1884$ (CO) $\mathrm{cm}^{-1}$. - ${ }^{1} \mathrm{H}$ NMR: $\delta=1.79\left(\mathrm{~m}, 8 \mathrm{H}, \mathrm{NCH}_{2} \mathrm{CH}_{2}\right)$, $3.28\left(\mathrm{~m}, 8 \mathrm{H}, \mathrm{NCH}_{2} \mathrm{CH}_{2}\right), 5.02\left(\mathrm{~s}, 5 \mathrm{H}, \mathrm{C}_{5} \mathrm{H}_{5}\right) .-{ }^{11} \mathrm{~B}$ NMR: $\delta=39.6(\mathrm{BCl}), 60.2(\mathrm{BW}) .-{ }^{13} \mathrm{C}\left\{{ }^{1} \mathrm{H}\right\}$ NMR: $\delta=25.46,25.76,26.36,26.75\left(\mathrm{NCH}_{2} \mathrm{CH}_{2}\right), 47.15,49.84$, 51.63, $55.76\left(\mathrm{NCH}_{2} \mathrm{CH}_{2}\right), 92.37\left(\mathrm{C}_{5} \mathrm{H}_{5}\right), 216.71,218.77$, $222.90(\mathrm{CO}) .-\mathrm{C}_{16} \mathrm{H}_{21} \mathrm{~B}_{2} \mathrm{ClN}_{2} \mathrm{O}_{3} \mathrm{~W}$ (530.28): calcd. C 36.24, H 3.99, N 5.28; found C 35.89, H 4.18, N 5.01.

10: $0.30 \mathrm{~g}(0.93 \mathrm{mmol})$ of $\mathbf{1}$ [4] is dissolved in $20 \mathrm{ml}$ of methanol and stirred for $1 \mathrm{~h}$ at ambient temperature. All volatiles are removed under high vacuum, and the residue is treated with of $40 \mathrm{ml}$ of hexane. After filtration $0.22 \mathrm{~g}(74.4 \%)$ of $\mathbf{1 0}$ is obtained at $-30{ }^{\circ} \mathrm{C}$ as a yellow solid. - IR (hexane): $\nu=1980,1921(\mathrm{CO}) \mathrm{cm}^{-1}$. ${ }^{1} \mathrm{H}$ NMR: $\delta=2.45,2.68,2.87,2.92\left(4 \mathrm{~s}, 12 \mathrm{H}, \mathrm{NMe}_{2}\right)$, $3.46(\mathrm{~s}, 3 \mathrm{H}, \mathrm{OMe}) 4.41\left(\mathrm{~s}, 5 \mathrm{H}, \mathrm{C}_{5} \mathrm{H}_{5}\right) .-{ }^{11} \mathrm{~B} \mathrm{NMR}$ : $\delta=34.4$ (BO), $69.0(\mathrm{BFe}) .-{ }^{13} \mathrm{C}\left\{{ }^{1} \mathrm{H}\right\}$ NMR: $\delta=34.17$, 38.94, 44.52, $48.41\left(\mathrm{NMe}_{2}\right), 53.65(\mathrm{OMe}), 85.14\left(\mathrm{C}_{5} \mathrm{H}_{5}\right)$, 218.06, 218.15 (CO). $-\mathrm{C}_{12} \mathrm{H}_{20} \mathrm{~B}_{2} \mathrm{FeN}_{2} \mathrm{O}_{3}$ (317.77): calcd. C 45.36, H 6.34, N 8.82; found C 44.98, H 6.24, N 8.37.

11: As described for 10, $0.38 \mathrm{~g}(0.87 \mathrm{mmol})$ of 4 [5] is dissolved in $20 \mathrm{ml}$ of methanol to give $0.23 \mathrm{~g}(68.0 \%)$ of 11 at $-30{ }^{\circ} \mathrm{C}$ as a yellow solid. - IR (hexane): $\nu=1981$, 1898, $1882(\mathrm{CO}) \mathrm{cm}^{-1} .{ }^{1}{ }^{1} \mathrm{H}$ NMR: $\delta=2.50,2.60,2.70$, $2.87\left(4 \mathrm{~s}, 12 \mathrm{H}, \mathrm{NMe}_{2}\right), 3.52(\mathrm{~s}, 3 \mathrm{H}, \mathrm{OMe}) 5.01(\mathrm{~s}, 5$ $\mathrm{H}, \mathrm{C}_{5} \mathrm{H}_{5}$ ). $-{ }^{11} \mathrm{~B}$ NMR: $\delta=33.7$ (BO), 67.1 (BMo). -
${ }^{13} \mathrm{C}\left\{{ }^{1} \mathrm{H}\right\}$ NMR: $\delta=34.36,39.01,42.63,47.97\left(\mathrm{NMe}_{2}\right)$, 54.09 (OMe), $92.64\left(\mathrm{C}_{5} \mathrm{H}_{5}\right), 226.07,226.71,234.14$ (CO).- $\mathrm{C}_{13} \mathrm{H}_{20} \mathrm{~B}_{2} \mathrm{MoN}_{2} \mathrm{O}_{4}$ (385.87): calcd. $\mathrm{C} 40.46, \mathrm{H}$ 5.22, N 7.26; found C 40.51, H 5.01, N 7.18.

12: As described for $\mathbf{1 0}, 0.54 \mathrm{~g}(1.68 \mathrm{mmol})$ of $\mathbf{1}$ [4] is dissolved in $20 \mathrm{ml}$ of ethanol to give an inseparable, solid mixture of 12 and $\left[\left\{\eta^{5}-\left(\mathrm{C}_{5} \mathrm{H}_{5}\right) \mathrm{Fe}(\mathrm{CO})_{2}\right\}_{2}\right]$. ${ }^{1} \mathrm{H}$ NMR: $\delta=1.19\left(\mathrm{t},{ }^{3} \mathrm{~J}=6.71 \mathrm{~Hz}, 3 \mathrm{H}, \mathrm{OCH}_{2} \mathrm{CH}_{3}\right)$, 2.46, 2.70, 2.89, $2.92\left(4 \mathrm{~s}, 12 \mathrm{H}, \mathrm{NMe}_{2}\right), 3.73$ (q, $\left.{ }^{3} J=6.71 \mathrm{~Hz}, 2 \mathrm{H}, \mathrm{OCH}_{2} \mathrm{CH}_{3}\right) 4.43\left(\mathrm{~s}, 5 \mathrm{H}, \mathrm{C}_{5} \mathrm{H}_{5}\right)$. $-{ }^{11} \mathrm{~B}$ NMR: $\delta=34.0(\mathrm{BO}), 69.1(\mathrm{BFe}) .-{ }^{13} \mathrm{C}\left\{{ }^{1} \mathrm{H}\right\}$ NMR: $\delta=18.48\left(\mathrm{OCH}_{2} \mathrm{CH}_{3}\right), 34.28,38.93,44.68$, $48.60\left(\mathrm{NMe}_{2}\right), 61.52\left(\mathrm{OCH}_{2} \mathrm{CH}_{3}\right), 85.14\left(\mathrm{C}_{5} \mathrm{H}_{5}\right), 218.13$, 218.24 (CO).

13: As described for $\mathbf{1 0}, 0.50 \mathrm{~g}(1.15 \mathrm{mmol})$ of 4 [5] is dissolved in $20 \mathrm{ml}$ of ethanol to give an inseparable, solid mixture of 12 and $\left[\eta^{5}-\left(\mathrm{C}_{5} \mathrm{H}_{5}\right) \mathrm{MoH}(\mathrm{CO})_{3}\right] .-{ }^{1} \mathrm{H}-\mathrm{NMR}$ : $\delta=1.20\left(\mathrm{t},{ }^{3} \mathrm{~J}=6.86 \mathrm{~Hz}, 3 \mathrm{H}, \mathrm{OCH}_{2} \mathrm{CH}_{3}\right), 2.51,2.62$, 2.71, $2.88\left(4 \mathrm{~s}, 12 \mathrm{H}, \mathrm{NMe}_{2}\right), 3.82\left(\mathrm{q},{ }^{3} J=6.86 \mathrm{~Hz}, 2\right.$ $\left.\left.\mathrm{H}, \mathrm{OCH}_{2} \mathrm{CH}_{3}\right)\right) 5.68\left(\mathrm{~s}, 5 \mathrm{H}, \mathrm{C}_{5} \mathrm{H}_{5}\right) .-{ }^{11} \mathrm{~B} \mathrm{NMR}: \delta=$ 33.4 (BO), 67.3 (BMo). $-{ }^{13} \mathrm{C}\left\{{ }^{1} \mathrm{H}\right\}$ NMR: $\delta=18.33$ $\left(\mathrm{OCH}_{2} \mathrm{CH}_{3}\right), 34.46,39.00,42.66,48.13\left(\mathrm{NMe}_{2}\right), 61.88$ $\left(\mathrm{OCH}_{2} \mathrm{CH}_{3}\right), 93.93\left(\mathrm{C}_{5} \mathrm{H}_{5}\right), 226.04,226.87,234.18(\mathrm{CO})$.

14: $0.64 \mathrm{~g}(1.48 \mathrm{mmol})$ of 4 is dissolved in $90 \mathrm{ml}$ of hex-

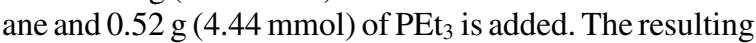
mixture is irridiated for $15 \mathrm{~h}$ at $-20{ }^{\circ} \mathrm{C}$ with a Heraeus TQ 150 high pressure mercury lamp. The solution is filtered, concentrated to $10 \mathrm{ml}$ in high vacuum and stored at $-30{ }^{\circ} \mathrm{C}$. $0.58 \mathrm{~g}(74.7 \%)$ of $\mathbf{1 4}$ is obtained as a yellow solid. - IR (toluene): $\nu=1932,1885,1853,1812(\mathrm{CO})$ $\mathrm{cm}^{-1} \cdot-{ }^{1} \mathrm{H}$ NMR: $\delta=0.82\left(\mathrm{dt},{ }^{3} J_{\mathrm{HH}}=7.60 \mathrm{~Hz},{ }^{3} J_{\mathrm{PH}}=\right.$ $\left.15.30 \mathrm{~Hz}, 9 \mathrm{H}, \mathrm{PCH}_{2} \mathrm{CH}_{3}\right), 1.41\left(\mathrm{dq},{ }^{3} J_{\mathrm{HH}}=7.60 \mathrm{~Hz}\right.$, $\left.{ }^{2} J_{\mathrm{PH}}=7.60 \mathrm{~Hz}, 6 \mathrm{H}, \mathrm{PCH}_{2} \mathrm{CH}_{3}\right), 2.70,2.81,2.97,3.08$ $\left(4 \mathrm{~s}, 12 \mathrm{H}, \mathrm{NMe}_{2}\right), 5.10$ (s (broad), $\left.5 \mathrm{H}, \mathrm{C}_{5} \mathrm{H}_{5}\right) .{ }^{11} \mathrm{~B} \mathrm{NMR}$ : $\delta=40.7$ (BBr), 68.8 (BMo). $-{ }^{13} \mathrm{C}\left\{{ }^{1} \mathrm{H}\right\}$ NMR: $\delta=7.94$ $\left(\mathrm{d},{ }^{2} \mathrm{~J}=2.20 \mathrm{~Hz}, \mathrm{PCH}_{2} \mathrm{CH}_{3}\right), 22.12\left(\mathrm{~d},{ }^{3} \mathrm{~J}=26.30 \mathrm{~Hz}\right.$, $\left.\mathrm{PCH}_{2} \mathrm{CH}_{3}\right), 39.32,42.25,42.96,48.46\left(\mathrm{NMe}_{2}\right), 92.32$ $\left(\mathrm{C}_{5} \mathrm{H}_{5}\right), 231.70\left(\mathrm{~d},{ }^{2} J=21.40 \mathrm{~Hz}, \mathrm{CO}\right), 224.30\left(\mathrm{~d},{ }^{2} J=\right.$ $21.90 \mathrm{~Hz}, \mathrm{CO}) .-{ }^{31} \mathrm{P}\left\{{ }^{1} \mathrm{H}\right\}(202.34 \mathrm{MHz}): \delta=52.8$ (s). $\mathrm{C}_{17} \mathrm{H}_{32} \mathrm{~B}_{2} \mathrm{BrMoN}_{2} \mathrm{O}_{2} \mathrm{P}$ (524.89): calcd. C 38.90, H 6.14, N 5.34; found C 38.56, H 6.20, N 5.06.

\section{Acknowledgements}

HB thanks DFG, FCI, RSoc and EPSRC for financial support. 
[1] For reviews on borane, boryl and borylene complexes see: H. Braunschweig, Angew. Chem. 110, 1882 (1998); G. I. Irvine, M. J. G. Lesley, T. B. Marder, N. C. Norman, C. R. Rice, E. G. Robins, W. R. Roper, G. R. Whittel, L. R. Wright, Chem. Rev. 98, 2685 (1998); H. Braunschweig, M. Colling, J. Organomet. Chem. 614, 18 (2000); H. Braunschweig, M. Colling, Coord. Chem. Rev. 223, 1 (2001), H. Braunschweig, in G. Meyer, D. Naumann, L. Wesemann (eds): Inorganic Chemistry Highlights, p. 213, Wiley-VCH, Weinheim, New York (2001).

[2] J. F. Hartwig, Xiaoming He, Angew. Chem. 108, 352 (1996) (for further examples see ref. [1]).

[3] A. Kerr, T. B. Marder, N. C. Norman, A. G. Orpen, M. J. Quayle, C. R. Rice, P. L. Timms, G. R. Whittell, J. Chem. Soc. Chem. Commun. 319 (1998).
[4] H. Braunschweig, B. Ganter, M. Koster, T. Wagner, Chem. Ber. 1291099 (1996).

[5] H. Braunschweig, M. Koster, R. Wang, Inorg. Chem. 38, 415 (1999).

[6] H. Braunschweig, M. Koster, J. Organomet. Chem. 588, 231 (1999);

[7] H. Braunschweig, M. Koster, K. W. Klinkhammer, Angew. Chem. 111, 2368 (1999).

[8] X. He, J. F. Hartwig, Organometallics, 15, 400 (1996).

[9] E. O. Fischer, W. Hafner, Z. Naturforsch. 10b, 140 (1955).

[10] H. Nöth, H. Schick, W. Meister, J. Organomet. Chem. 2, 401 (1964).

[11] P. P. Power, A. Moezzi, M. M. Olmstead, J. Chem. Soc. Dalton Trans. 2429 (1992).

[12] H. Nöth, M. Wagner, Chem. Ber. 124, 1963 (1991). 